\title{
Status Of THE NASA Micro PUlSe LiDAR Network (MPLNET): OVERVIEW OF THE NETWORK AND FUTURE PLANS, NEW VERSION 3 DATA PRODUCTS, AND THE POLARIZED MPL
}

\author{
Ellsworth J. Welton ${ }^{1}$, Sebastian A. Stewart ${ }^{2}$, Jasper R. Lewis ${ }^{3}$, Larry R. Belcher ${ }^{2}$, James R. \\ Campbell ${ }^{4}$, and Simone Lolli ${ }^{3}$ \\ ${ }^{I}$ NASA Goddard Space Flight Center, USA. *Ellsworth.J.Welton@nasa.gov \\ ${ }^{2}$ Science Systems and Applications Inc., Goddard Space Flight Center, USA \\ ${ }^{3}$ University of Maryland Baltimore County, Goddard Space Flight Center, USA \\ ${ }^{4}$ Naval Research Laboratory, Marine Meteorology Division, USA
}

\begin{abstract}
The NASA Micro Pulse Lidar Network (MPLNET) is a global federated network of Micro-Pulse Lidars (MPL) co-located with the NASA Aerosol Robotic Network (AERONET). MPLNET began in 2000, and there are currently 17 long-term sites, numerous field campaigns, and more planned sites on the way. We have developed a new Version 3 processing system including the deployment of polarized MPLs across the network. Here we provide an overview of Version 3, the polarized MPL, and current and future plans.
\end{abstract}

\section{INTRODUCTION}

The NASA Micro-Pulse Lidar Network (MPLNET) [1] is a global federated network of polarized Micro-Pulse Lidar (MPL) systems designed to measure aerosol and cloud vertical structure continuously, over long time periods required to contribute to climate change studies and provide ground validation for satellite sensors and related aerosol modeling and forecasting efforts. MPLNET began in 2000, and there are currently 17 long-term and numerous short-term field campaign sites. Seven of the long-term sites have $10+$ years of data, and many more have 5+ years. There are 6 more sites in planning stages towards operational status by end of 2017 and several more at proposal stage. Most MPLNET sites are co-located with the NASA Aerosol Robotic Network (AERONET) [2], producing both column and vertically resolved data on aerosol and cloud properties and the evolution and structure of the planetary boundary layer (PBL). MPLNET is also a member of the WMO Global Atmospheric Watch (GAW) Aerosol Lidar Observation Network (GALION) [3].
MPLNET has been running the Version 2 processing system [4] since 2006. We have developed an enhanced and more mature Version 3 (V3) processing system, set for release in spring 2017. The new website is already active at http://mplnet.gsfc.nasa.gov. V3 introduces a new polarized MPL, already deployed throughout the network. In addition to the new polarized data products, vast improvements to all products will be provided including new cloud optical data and a more robust mixed layer depth product to examine the PBL. The entire V2 data archive is available from the new V3 website, and new V2 data will continue to be produced through 2017. This presentation will provide an overview of MPLNET Version 3, the polarized MPL, and current and future plans.

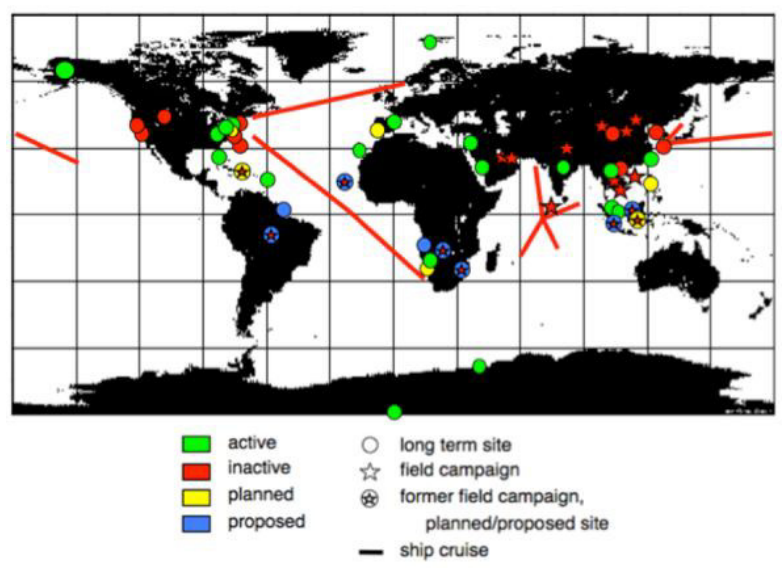

Figure 1. MPLNET Sites and Deployments

\section{THE POLARIZED MPL}

The original MPL design from the 1990s was depolarized. A solution to polarize the MPL was 
presented by Flynn et al [5] in the early 2000s, and many instruments were sold by the manufacturer (Sigma Space Corporation). This version of the polarized MPL was not used within MPLNET because a full performance and sensitivity study was not completed. The design showed promise but was not ready to integrate into MPLNET. In addition, the design utilized a liquid crystal retarder (LCR) to switch polarization states, but the nematic LCR is limited to millisecond switching speeds. In practice the MPL was switched much slower which could affect cloud observations.

Several years ago, the MPLNET team began working with Sigma Space to redesign the polarized MPL to utilize a ferroelectric liquid crystal (FLC) rotator, which is capable of switching the emitted polarization state of the transmitted MPL pulses at microsecond data rates. The new design produces similar data, and data processing techniques described by Flynn et al [5] remain applicable. However, the operation of the MPL using a rotator versus a retarder requires a different assembly methodology and calibration process. Stewart et al [6] (this issue) present an overview of the new polarized MPL design and present a methodology for calibrating the FLC component. A more thorough presentation of this polarized MPL, its performance characteristics, system bias, and calibration procedures will be provided in a forthcoming publication.

In summary, the new calibration protocols account for the optical properties of the MPL components, the most critical being the polarizing beam-splitter (PBS) and the FLC. The window used for data collection may also be important if stressed due to mechanical/temperature fluctuations. The diattenuation, retardance, and alignment/rotation angles of the PBS and FLC are critical parameters. Once obtained (see [6]), the MPL can be calibrated to reduce systematic bias in the measured signals and resulting volume depolarization ratio to within fractions of a percent. As a result, the new polarized MPL is capable of measuring the molecular volume depolarization background with sufficient time averaging to reduce signal noise $(\sim 1$ hour at night for less turbid conditions). Without careful application of such calibrations, large systematic bias (up to $\sim 30 \%$ ) may be present in the signals, especially for returns from weakly depolarizing layers (e.g. molecular background). The affects are highly non-linear, with low bias in signals from strongly depolarizing layers. Thus the new MPL is easily capable of detecting the presence of non-spherical particles (dust, ice clouds), but quantitative analysis requires the polarization calibrations. Figure 2 presents examples of the new MPL signals and depolarization ratios measured at NASA Goddard Space Flight Center from May 20 - 24, 2015.

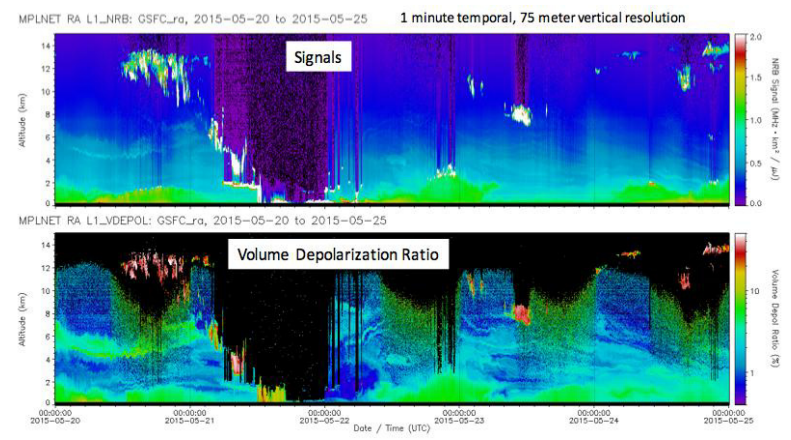

Figure 2. MPLNET V3 Level 1 Products

In addition to calibrating the optical components, ferroelectric materials are temperature dependent by nature. The FLC is subject to both solar background and internal heat within the MPL, which can change the location of the fast axes, and the resulting rotation angle (studies indicate approximately $0.3^{\circ}$ change per $\left.1^{\circ} \mathrm{C}\right)$. A temperature correction has been developed as part of the new calibration procedures, but is a second order affect unless the FLC temperature changes by several degrees (this is uncommon). For typical operating temperatures, the expected bias from temperature dependent FLC effects is $\sim 0.5 \%$ at most. The temperature calibrations are more time consuming and difficult to determine and apply per MPL. If the basic polarization component calibrations are applied to the MPL, but the temperature dependent effects are ignored, then for normal operating temperatures it is possible to accurately measure volume depolarization ratios $\geq$ $1.5 \%$. For more weakly depolarizing aerosols (such as sulfate), the temperature corrections must be applied to produce quality data with minimum of systematic bias. 


\section{VERSION 3 PROCESSING SYSTEM}

The new V3 processing system is a completely new version of MPLNET. In addition to the new polarized MPL, all data communications, archiving, processing code, and the website are new. Some V3 algorithms are based on mature V2 processing, but the code and processing structure are new. Some of the important new changes in V3 include:

- CF compliant NETCDF4 data formats

- QA flags at all data levels

- New product structure and definitions

- NASA GEOS-5 Meteorology

- Online data ordering and delivery

- Near-real-time (NRT) Level 1.5 products are now quality assured

- Custom data products and delivery to aerosol forecasting centers

Both V2 and V3 data will be provided from the new website. Older V2 data will be provided in the new V3 data file formats. Table 1 provides definitions and descriptions of the new V3 products levels.

Signal data (NRB) are processed using mature V1 and V2 algorithms [7,8]. The new polarized MPL and calibration requirements will be presented in a future paper. The V3 cloud products (CLD) are new, and generated from algorithms presented by Lewis et al [9], including improved cloud detection versus V2 and new thin cloud optical depth and phase information. The new V3 planetary boundary layer products (PBL) include mixed layer depths presented by Lewis et al [10]. Finally, the aerosol algorithms remain unchanged from V2 [11, 12], the only differences are in the operational implementation (online algorithm theoretical basis documents, ATBD, will be available on our website soon).

L1 products are automated and generated in NRT (latency for most all of network is $\sim 1.5$ hours). L1 processing will utilize forecast meteorology from the NASA GEOS-5 model [15]. Quality assurance screens will be applied to all L15 data. In V2, this was only done for L2 products. This change follows the same protocol developed for AERONET V3 data. L15 processing will also occur in NRT and browse imagery will be available on our website, but data files will not be publicly downloadable until the final assimilated GEOS-5 data are available for reprocessing (typically $\sim 24$ hour delay). The NRT L15 data files will be made available to operational aerosol forecasting centers under agreement.

Table 1. V3 Product Level Definitions

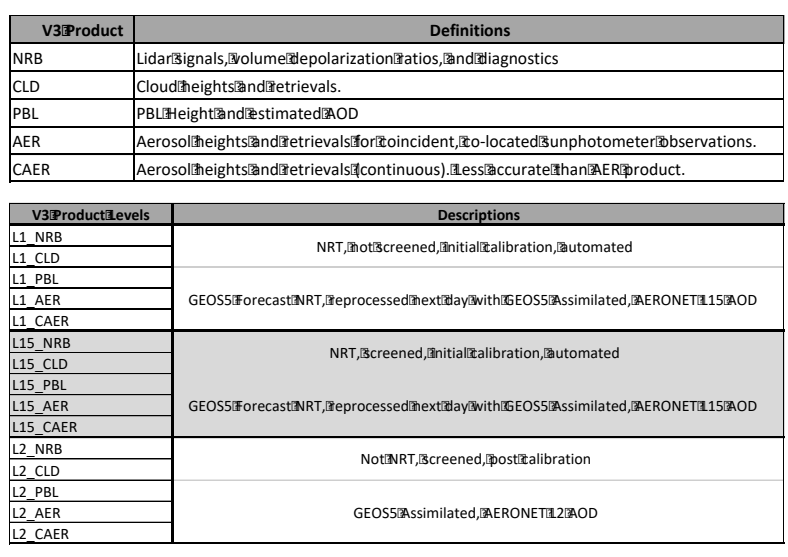

Figure 3 presents examples of V3 data products retrieved from data collected at NASA Goddard Space Flight Center for the same time span as shown in Figure 2 (May $20-24,2015$ ). The top pane displays V3 cloud heights (gray) and identification of ice clouds (purple). The next pane presents estimates of thin cloud optical depth from [9], where the results are bounded using lidar ratios of 20 and 30 sr (dark and light blue respectively). The following pane shows retrievals of aerosol extinction [11,12] and an overlay of the new V3 mixed layer depth (brown). The bottom pane displays the column aerosol optical depth (AOD), from AERONET (green), the continuous V3 aerosol product (CAER, red), and the estimate of the PBL AOD (brown). All MPLNET aerosol retrievals are constrained by the co-located AERONET data [11].

\section{CONCLUSIONS}

An overview of the new V3 MPLNET processing system has been presented. V3 data products will be released in 2017, and joint processing of V2 data will continue through at least 2017. All V2 products will be available from the new website, including after joint processing of V2 ends. The size and distribution of the network and the planned addition of new sites in the near term 
were shown. Additional growth of the permanent, long-term sites in the network requires a funding increase to accommodate new personnel. However, MPLNET will continue to participate in short-term field campaigns worldwide as well as the WMO GALION project.

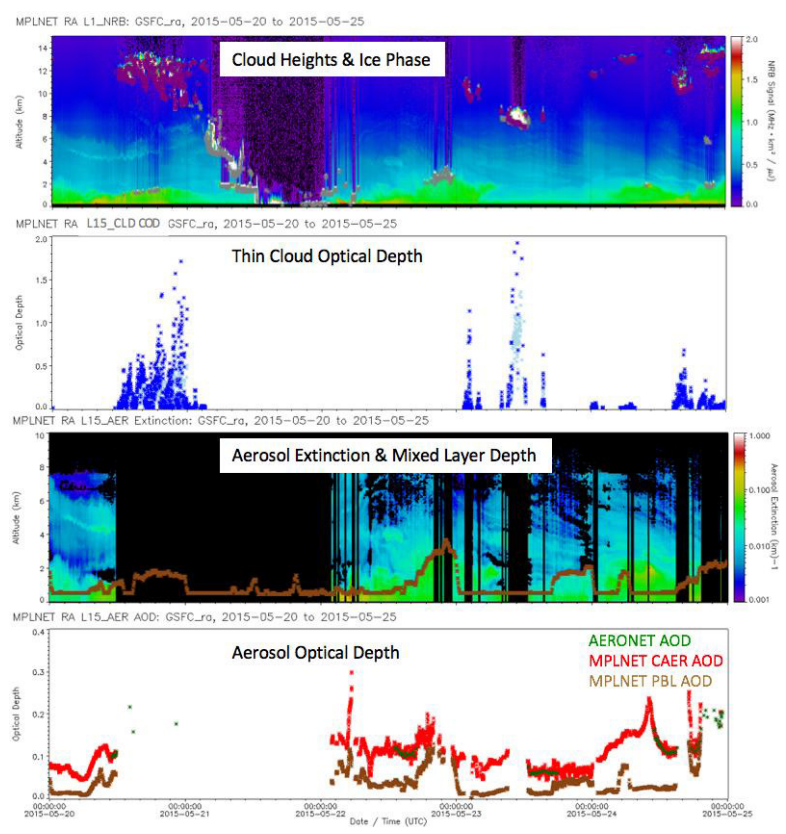

Figure 3. MPLNET V3 Cloud, Aerosol and PBL Products

\section{ACKNOWLEDGEMENTS}

The MPLNET project is funded by the NASA Radiation Sciences Program and Earth Observing System. We also thank Justin Fisher, Adam Slagel, and Phillip DeCola at Sigma Space Corporation for helpful collaborations developing and testing the new polarized MPL.

\section{References}

[1] Welton, E. J., et al, 2001: Global monitoring of clouds and aerosols using a network of micropulse lidar systems, in Lidar Remote Sensing for Industry and Environmental Monitoring, U. N. Singh, T. Itabe, N. Sugimoto, (eds.), Proc. SPIE, 4153, 151-158.

[2] Holben B.N., et al, 1998: AERONET - A federated instrument network and data archive for aerosol characterization. Rem. Sens. Environ., 66, 1-16.
[3] Bosenberg, J., et al, 2007: Plan for the implementation of the GAW Aerosol Lidar Observation Network GALION. WMO GAW Report No. 178, WMO/TD-No. 1443.

[4] Welton E.J., et al., 2010: The NASA Micro Pulse Lidar Network (MPLNET): summary of the last 10 years, current status, and future plans, Proceedings of the 25th Inter. Laser Radar Conf., 1, 875-878.

[5] Flynn, C., et al, 2007: Novel polarizationsensitive micropulse lidar measurement technique. Optics Express, 15, 2785-2790.

[6] Stewart, S.A., et al, 2017: Experimental Characterization of the Ferroelectric Liquid Crystal (FLC) Modulator in the Polarized Micro Pulse Lidar. Proceedings of the 28th Inter. Laser Radar Conf., this issue.

[7] Campbell, J.R., et al, 2002: Full-time, Eye-Safe Cloud and Aerosol Lidar Observation at Atmospheric Radiation Measurement Program Sites: Instrument and Data Processing. J. Atmos. Oceanic Technol., 19, 431-442.

[8] Welton, E.J., and J.R. Campbell, 2002: Micropulse Lidar Signals: Uncertainty Analysis. J. Atmos. Oceanic Technol., 19, 2089-2094.

[9] Lewis, J.R., et al, 2016: Overview of MPLNET Version 3 Cloud Detection. J. Atmos. Oceanic Tech., 33, 2113 - 2134, doi: 10.1175/JTECHD-15-0190.1.

[10] Lewis, J.R., et al, 2013: Improved boundary layer depth retrievals from MPLNET, J. Geophys. $\quad$ Res., 118, 9870-9879, doi:10.1002/jgrd.50570.

[11] Welton, E.J., et al, 2000: Ground-based Lidar Measurements of Aerosols During ACE-2: Instrument Description, Results, and Comparisons with other Ground-based and Airborne Measurements. Tellus B, 52, 635-650.

[12] Welton, E.J., et al, 2002: Measurements of aerosol vertical profiles and optical properties during INDOEX 1999 using micro-pulse lidars. J. Geophys. Res., 107, 8019, doi:10.1029/2000JD000038. 